\title{
Use of mitral homograft to support a mechanical valve prosthesis: A feasible solution for recurrent mitral valve dysfunction
}

\author{
José M. Bernal, MD, ${ }^{\mathrm{a}}$ Francisco Gutiérrez, MD, ${ }^{\mathrm{a}}$ M. Carmen Fariñas, MD, ${ }^{\mathrm{b}}$ Elena Arnaiz, MD, ${ }^{\mathrm{a}}$ Carmen Diago, MD, \\ Alejandro Pontón, MD, ${ }^{a}$ Blanca Ruiz, MD, ${ }^{\mathrm{d}}$ J. Fernando Val-Bernal, MD, ${ }^{\mathrm{c}}$ and José M. Revuelta, MD, ${ }^{\mathrm{a}}$ Santander, Spain
}

We describe the case of a man with recurrent prosthetic mitral valve dysfunction that was resolved indirectly with a mitral valve homograft.

\section{CLINICAL SUMMARY}

A 54-year-old man with a diagnosis of mitroaortic endocarditis caused by methicillin-sensitive Staphylococcus epidermidis underwent mitral and aortic valve replacement (CarboMedics $31 \mathrm{~mm}$ and $23 \mathrm{~mm}$; CarboMedics Inc, Austin, Tex) and received cloxacillin benzathine (INN cloxacillin) and gentamicin for 7 days, followed by cloxacillin for 5 weeks. Results of valve cultures and control blood cultures at 15 and 60 postoperative days were negative. Six months later, a first reoperation was performed because of endocarditis of both prostheses. An aortic homograft substituting the aortic root and a new CarboMedics prosthesis were implanted. Results of valve cultures, blood cultures with prolonged incubation, and serologic tests for Coxiella, Brucella, Mycoplasma, and Bartonella infections were negative. Combined treatment with vancomycin, gentamicin, and rifampin (INN rifampicin) for 2 weeks was administered, followed by vancomycin and rifampin for 4 weeks. Results of blood cultures were negative. Six months later, a second reoperation was needed because of dehiscence of the mitral prosthesis in the mitroaortic junction and macroscopic appearance of endocarditis. A new CarboMedics prosthesis was inserted. Vancomycin and rifampin were empirically administered during 6 weeks and gentamicin during 2 weeks. All cultures and serologic tests were unrevealing. Three months later, a third prosthetic valve replacement for infective endocarditis was performed. Results of cultures and serologic tests were negative. The patient received linezolid and rifampin for 6 weeks because of poor tolerance of vancomycin.

Six months later, the patient underwent a fourth reoperation, this time for acute pulmonary edema and severe mitral

\footnotetext{
From the Department of Cardiovascular Surgery, ${ }^{a}$ the Infectious Diseases Unit, Department of Internal Medicine, ${ }^{\mathrm{b}}$ the Department of Pathology, ${ }^{\mathrm{c}}$ and the Department of Cardiology, ${ }^{\mathrm{d}}$ Hospital Universitario Valdecilla, Universidad de Cantabria, Santander, Spain.

Received for publication Nov 14, 2007; revisions received Dec 7, 2007; accepted for publication Dec 12, 2007.

Address for reprints: José M. Bernal, MD, Department of Cardiovascular Surgery, Hospital Universitario Valdecilla, E-39008 Santander, Spain (E-mail: bernal@ humv.es).

J Thorac Cardiovasc Surg 2009;137:762-3

0022-5223/\$36.00

Copyright (c) 2009 by The American Association for Thoracic Surgery doi:10.1016/j.jtcvs.2007.12.082
}

insufficiency caused by dehiscence of the mitral prosthesis. Results of cultures and serologic tests were negative. Signs of endocarditis at gross intraoperative inspection were not observed. The prosthesis was not lined with endocardium and showed dehiscence at various sites, particularly in the mitroaortic junction. When the prosthetic ring was removed, the native mitral ring had a raw flesh appearance, with suggestive signs of rejection or intolerance to the Dacron polyester fabric of the prosthetic suture ring. A complete mitral homograft was implanted, with several 4-0 polypropylene sutures used to fix the graft's papillary muscles to the native papillary projections. The graft annulus was fixed to the recipient annulus with a running suture (3-0 polypropylene). The annular suture was supported with a 28-mm CarboMedics AnnuloFlex annuloplasty ring (4 $\mathrm{mm}$ smaller diameter than the measured ring). A possible anterior paracommissurotomy leak was repair with the Alfieri procedure. After discontinuation of cardiopulmonary bypass, transesophageal echocardiography showed severe residual mitral regurgitation (grade 3/4). Under cardiopulmonary bypass, the prosthetic ring was removed, and a mechanical CarboMedics 29-mm prosthesis was implanted inside the mitral homograft with a technique similar to transvalvular mitral prosthesis implantation preserving the entire native valve apparatus. ${ }^{1}$ Methicillin-sensitive $S$ epidermidis was isolated in the culture of the prosthetic valve. Results of blood cultures and serologic tests were negative. Results of polymerase chain reaction amplification followed by direct sequencing to identify microbial pathogens in the tissue from mitral and aortic valves were negative. Histopathologic examination of a thrombus over the annulus of the mechanical prosthetic valve revealed a predominantly eosinophilic infiltrate without bacteria. Results of skin tests to suture threads and prosthetic ring material were negative. Combined treatment with cloxacillin, rifampin, and gentamicin was given for 2 weeks, followed by cloxacillin and rifampin for 5 weeks. Before the patient's discharge from the hospital, transesophageal echocardiography showed residual mitral regurgitation (grade 2/ 4). Currently, 14 months after implantation of a mechanical prosthesis inside a supporting mitral valve homograft, the patient is free of symptoms with mild regurgitation (grade 1/4; Figure 1).

\section{DISCUSSION}

The prognosis of patients with recurrent mitral valve dysfunction undergoing one or more reoperations for 


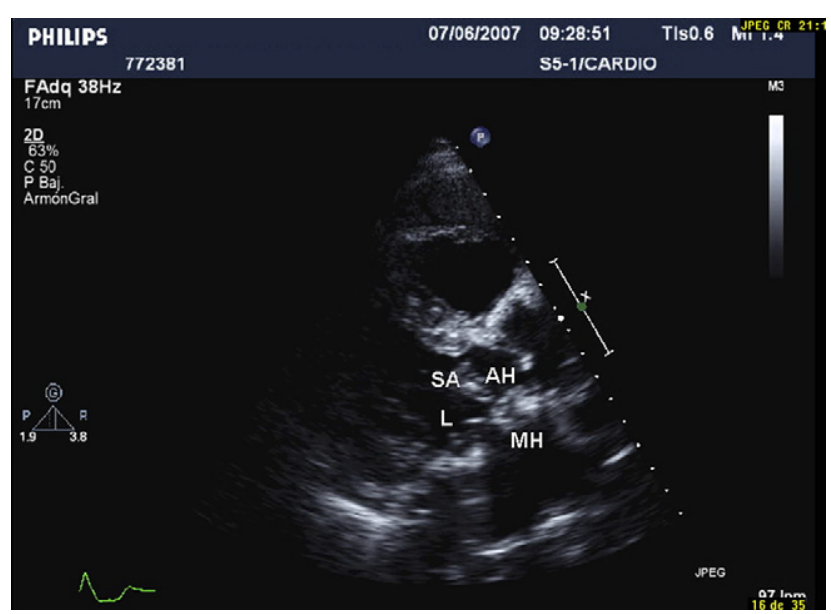

FIGURE 1. Transthoracic diastolic long-axis view. $S A$, Mitral homograft subvalvular apparatus with papillary muscle and chordae tendineae; $A H$, aortic homograft; $L$, mechanical mitral prosthesis leaflets; $M H$, mitral homograft.

substitution of the prosthesis is very poor. ${ }^{2-4}$ In our case, four consecutive reoperations were needed because of recurrent prosthetic dehiscence and mitral valve dysfunction. It was considered that a mitral homograft could be a valid alternative, but functional results were disappointing because of limited mobility and deficient coaptation of the anterior leaflet. Implantation of an annuloplasty ring and repair of the valve commissure were unsuccessful, and instead of explanting the homograft it was decided to remove the annuloplasty ring and insert a bioprosthesis in a transvalvular position, with the homograft acting as a native valve. At 14 months of follow-up, the patient is free of symptoms, and functional results are satisfactory. Hypersensitivity to the suture ring of the valve prosthesis is an appealing explanation for our findings. In this respect, the interposed homograft avoided the potential risk of intolerance to the suture ring and also prevented direct contact between the infected tissue and the prosthesis.

We thank Marta Pulido, MD, for editing the manuscript and for editorial assistance.

\section{References}

1. Revuelta JM, Bernal JM, Rabasa JM. Transvalvular technique for implantation of a mitral valve homograft. J Thorac Cardiovasc Surg. 1996;111:281-2.

2. Echevarria JR, Bernal JM, Rabasa JM, Morales D, Revilla Y, Revuelta JM. Reoperation for bioprosthetic valve dysfunction. A decade of clinical experience. Eur $J$ Cardiothorac Surg. 1991;5:523-7.

3. Pérez-Vázquez A, Fariñas MC, García-Palomo JD, Bernal JM, Revuelta JM, González-Macías J. Evaluation of the Duke criteria in 93 episodes of prosthetic valve endocarditis: could sensitivity be improved? Arch Intern Med. 2000;160:1185-91.

4. Fariñas MC, Pérez-Vázquez A, Fariñas-Alvarez C, García-Palomo D, Bernal JM Revuelta JM, et al. Risk factors of prosthetic valve endocarditis: a case-control study. Ann Thorac Surg. 2006;81:1284-90.

\section{Concomitant giant coronary artery and coronary sinus aneurysms}

Basar Sareyyupoglu, MD, James E. Davies, MD, Grace Lin, MD, and Thoralf M. Sundt, MD, Rochester, Minn

Coronary artery aneurysms are uncommon, being noted in $0.15 \%$ to $4.9 \%$ of patients undergoing coronary angiography. Only a small fraction of these aneurysms are of sufficient size to be considered "giant," a term commonly applied when such lesions exceed 20 to $50 \mathrm{~mm}$ in diameter. ${ }^{1,2}$ Coronary artery fistulae are equally rare, reportedly occurring in $0.22 \%$ of patients undergoing cardiac catheterization. $^{3}$ Curiously, the former lesion has been reported

\footnotetext{
From the Cardiovascular Surgery and Cardiovascular Diseases Departments, Mayo Clinical, Rochester, Minn.

Received for publication Nov 13, 2007; accepted for publication Oct 27, 2008.

Address for reprints: Thoralf M. Sundt, MD, Mayo Clinic, Cardiothoracic Surgery Department, Joseph 5-200, 200 First St SW, Rochester, MN 55905 (E-mail: sundt.thoralf@mayo.edu).

J Thorac Cardiovasc Surg 2009;137:763-5

$0022-5223 / \$ 36.00$

Copyright (C) 2009 by The American Association for Thoracic Surgery

doi:10.1016/j.jtcvs.2008.10.021
}

associated with the latter in $30 \%^{2}$ to $80 \%{ }^{1}$ of cases, with communication most often to the left ventricle, ${ }^{1}$ right ventricle, or right atrium. ${ }^{2}$ One such fistula with associated dilatation of the right coronary artery (RCA) but without frank aneurysm has been reported to the coronary sinus (CS). ${ }^{4}$ We recently encountered a case of concomitant giant aneurysms of the proximal RCA and CS, with a distal RCA-CS fistula.

\section{CLINICAL SUMMARY}

An 82-year-old woman was admitted to the hospital with worsening right heart failure. Three years previously, she was incidentally discovered to have a giant RCA aneurysm. Surgical repair was declined at that time by the patient. Because of worsening symptoms of dyspnea on exertion and pedal edema, however, the patient sought evaluation at our institution. She denied angina pectoris. 